\title{
Building Resilience in Temporary Organizations: Lessons from a Shipyard
}

\author{
Anne Russel, Stéphanie Tillement, and Benoit Journé
}

\section{INTRODUCTION}

This chapter discusses the organizational and occupational dimensions of sustained reliable performance (Perrow, 2011) in temporary organizing contexts. Studying organizations through a temporary lens (Söderlund, 2000) has enabled the identification of clear differences between permanent organizations, mainly characterized by "production processes and continual development" (Lundin \& Söderholm, 1995, p. 439), and temporary organizations, characterized by having a predetermined lifetime. Temporary organizations have been defined as new forms of organizations that adapt to deal with new "problems concerning the complexity
A. Russel $(\bowtie) \cdot S$. Tillement · B. Journé
Département SSG, IMT Atlantique, Nantes, France
e-mail: anne.russel@imt-atlantique.fr
S. Tillement
e-mail: stephanie.tillement@imt-atlantique.fr
B. Journé
e-mail: benoit.journe@univ-nantes.fr

(C) The Author(s) 2022

R. Pinheiro et al. (eds.), Towards Resilient Organizations and Societies, Public Sector Organizations, https://doi.org/10.1007/978-3-030-82072-5_4 
and the uncertainty of the task, the complexity among the interdependent activities, the task's uniqueness, the lack of standardized procedures and the temporary nature of the task" (Söderlund, 2000, p. 64). Thus, it seems that temporary organizations and temporary forms of organizing are an appropriate solution for dealing with organizational complexity.

The aforementioned authors also show that complexity presents a risk for organizational performance and can impact the organization's ability to adapt to a frequently changing environment or to a new industrial tempo. In our case, we suggest switching from a temporary organization perspective (often restricted to project-based organizations in the project management literature) to the more dynamic approach of temporary organizing. We define organizing as the emergent process of production of an organization by the organizational actors and through the reflectivity of actors (Weick, 1979).

Other authors emphasize the safety issues that stem from organizational complexity. Indeed, normal accident theory (NAT) (Perrow, 2011 ) and theory about high reliability organizations (HROs) (Weick \& Roberts, 1993) show how major accidents can be caused by complexity. NAT demonstrates that complex and tightly coupled systems, such as in nuclear power plants, are exposed to a high level of risk and will inevitably have accidents. Perrow shows that, in the case of the Three Mile Island accident, it was the characteristics of the system itself that made the accident inevitable.

In contrast, the resilience engineering perspective (Furuta, 2015; Hollnagel et al., 2006) views resilience as part of a systemic process which aims to prevent major accidents from happening: "From a systemic view, resilience is the intrinsic ability of a system to adjust its functioning prior to, during, or following changes and disturbances, so that it can sustain required operations under both expected and unexpected conditions" (Furuta, 2015, p. 446). The different conceptions of resilience that are held by the resilience engineering theorists and the high reliability theorists emphasize the importance of its temporal dimension. High reliability theorists seek to understand the principles that organizations rely on to manage the unexpected: while developing a very dynamic approach, they oppose the anticipation of events to their containment. Resilience is one principle that can be used to contain unexpected events when they occur and is therefore associated with re-activeness. In their view, resilience is mainly an ex-post strategy. Hollnagel et al. (2006) and Furuta (2015) 
adopt a more holistic perspective of resilience which encompasses anticipation and therefore goes beyond the dichotomy between ex-ante and ex-post dimensions of resilience. In this chapter, we adopt the resilience engineering perspective, which encompasses what happens before, during and after unexpected events. More precisely, we adopt Hollnagel's vision of resilience as enabling safety by "looking at what goes right" rather than "what goes wrong" (Hollnagel, 2016, p. 189).

It therefore seems that, when dealing with issues related to high levels of safety in complex settings, it is longstanding organizations with strong organizational routines that offer the most appropriate forms of organizing. However, few researchers have looked at how actors in temporary organizing contexts, where routines and habits are not shared by all members of the organization, enhance and sustain resilience when facing uncertainty in safety-critical contexts (Saunders, 2015; Saunders et al., 2016). For instance, in complex projects, where work is always singular and is distributed between several companies and occupations, a common organizational safety culture or individual sensemaking appear insufficient to support reliable performance.

This chapter addresses this gap in the literature by demonstrating that temporary organizations can also use resilient mechanisms to deal with major safety issues, and that temporary forms of organizing can help complex projects to be efficiently and safely carried out. In this perspective, we define project resilience as the ability of a project to prevent major accidents from happening while maintaining its intended level of industrial performance. We examine this proposition by studying the case of an inter-organizational and safety-critical project: the construction by a shipyard of a series of ships. This project is managed by a public organization but involves many private contractors and a wide array of occupations. Thus, inter-organizational and inter-occupational coordination are crucial for reliable performance. As a temporary organization which must ensure a high level of safety while maintaining performance in an uncertain situation, under time pressure, this case is particularly relevant for enriching the literature on project resilience.

In such temporary settings, where permanent and more temporary forms of organizing must be coordinated, we argue that the occupational dimension is essential to enhancing resilience and sustaining reliable performance. Looking at the meso-level, i.e. the professional occupations involved in the project, we question how temporary forms of organizing and occupational groups together contribute to the resilience of the whole 
project. We show that the ability of the project to coordinate temporary organizing forms is key to achieving (safe) performance.

In HROs, safe performance is achieved by developing a strong organizational safety culture that is shared by all members of the organization. By contrast, we show that in complex and temporary projects, safe performance is achieved by articulating a wide variety of organizational actors who do not share the same occupational cultures and habits and who become involved for different periods of time. Building on the concept of communities of practice developed by Lave and Wenger (Lave, 1991; Lave \& Wenger, 1991) and on the articulation work literature, we show that the articulation of work between various levels of participation helps the project to be more efficient and to ensure occupational and organizational safety, highlighting the link between high-quality activity and the safety of future users of the ships.

The chapter discusses the mechanisms and conditions that contribute to organizational resilience in temporary organizations. Through the study of a construction project that has to deal with complexity and safety issues, we demonstrate the key roles that temporary organizing and occupational communities play in the project's resilience. More precisely, this chapter addresses the following questions: How is resilience expressed in temporary organizations? Can temporary forms of organizing be compatible with sustainable occupational expertise? Under which conditions can temporary and more permanent forms of organizing be coordinated to ensure safe organizational performance?

By studying the case of a specific occupational group-the boilermakers-we show how they prevent major accidents by continually anticipating, adapting and reacting to normally disturbed situations. Taking an ethnographic approach (Garfinkel, 1967; Van Maanen, 1979) based on the observation of the routine daily activity of the field actors, we highlight that in complex projects resilience is first enhanced by the use of temporary organizing forms that provide greater flexibility and help the project to adapt to a discontinuous production flow. We then demonstrate that resilience is also built at an occupational level. Adopting an occupational lens (Brown \& Duguid, 1991; Gherardi, 2018) shows the extent to which resilience, seen as the situated ability to anticipate and adapt to safety issues, is embedded in a long-term trajectory. The occupation thus appears to enable the existence of a common set of values and principles around which workers belonging to various companies and working under different contracts can come together. Finally, we 
demonstrate that the project's resilience is conditioned by the ability of the project's management to coordinate different expertise and levels of participation. The chapter concludes by discussing the implications of considering resilience in temporary organizing contexts.

\section{ENSURING Resilience IN TEMPORARY Organizing ConteXts}

Unlike permanent organizations, which have stable processes and personnel that enable enduring work routines and knowledge to be developed, temporary organizations "bring together a group of people that are unfamiliar with one another's skills, but must work interdependently on complex tasks" (Bechky, 2006, p. 3). Söderlund (2000) proposes a typology of permanent and temporary forms of organizing which are categorized according to two main criteria: the structure (permanent or temporary) and the type of participation (permanent or temporary). This typology gives rise to four forms of organizing: temporary organizing (temporary structure and temporary participation); project organizing (temporary structure and permanent participation); temporary employment (permanent structure and temporary participation); and permanent organizing (permanent structure and permanent participation). So, in this first perspective, an organization that corresponds to one of these four organizational forms can be considered as temporary since either its participation or its structure has temporary aspects.

Another view of temporary organizations proposes taking a more processual perspective, which focuses on the role of the "individual and collective agents" (Bakker et al., 2016, p. 1708) and considers the structural dimension as an evolving rather than a stabilized component. Consequently, in adopting a processual lens, these authors use the term organizing instead of organization to show the constantly evolving nature of the organizational forms studied. Therefore, for them, clear differentiation between permanent and temporary organizing may not be relevant. Rather, they consider that "in temporary organizing, what is permanent and what is temporary are sometimes fuzzy and often intertwined" (Bakker et al., 2016, p. 1708), and they contend that temporary organizing should be understood as a complex mix of temporary and permanent elements. This second definition of temporary organizing proposes a deeper understanding of the phenomenon and encourages further studies to analyse more accurately the dynamics involved (Bakker et al., 2016). 
In this chapter, we adopt a processual view of forms of temporary organizing to study how the permanent and temporary dimensions are effectively intertwined and how this contributes to project resilience. Indeed, temporary organizing can be considered to be a mechanism for resilience that is employed by certain organizations when facing normally disturbed situations and evolving in more dynamic and changing contexts. This enables the mobilization of a more agile workforce (outsourcing, contracting, etc.) and of more adaptive structures (group projects), oriented towards shorter-term tasks. Temporary organizing is therefore a way of dealing with organizational complexity, such as that relating to the uniqueness of tasks or the interdependence of activities (Söderlund, 2000).

Interestingly, this stream of the literature seems to associate temporary organizing with support for resilience, whereas researchers who have studied HROs consider that it is the permanent nature of organizations that supports resilience. Indeed, according to high reliability theorists, the success of HROs in ensuring continuous high levels of safety while constantly experiencing high levels of technological risk and unpredictable events is partly explained by the fact that they have developed a strong organizational safety culture and strategy (Milch \& Laumann, 2016). Weick and Roberts (1993) emphasize the roles of individual mindfulness and heedful interacting as pillars of resilience in highly disturbed contexts. More recently, Weick and Sutcliffe (2015) put forward a set of principles that support high reliability through anticipating the unexpected events and reacting to them once they have occurred.

In line with the situated and dynamic organization approaches, we therefore focus on the organizational, interactional and individual mechanisms that constitute pillars of resilience in temporary organizing contexts. We draw our inspiration from the five pillars of reliability defined by HRO researchers (Weick \& Sutcliffe, 2015) while adhering to Hollnagel's concept of resilience, which includes strategies for anticipating and adapting to unexpected events (Hollnagel, 2016). Saunders (2015) is one of the few authors who has considered how to apply HRO principles to safety-critical projects and remarks that, in the case of temporary projects, "these [high reliability] practices were often fragile, with much depending on the tenacity and strength of will of individual project managers rather than being embedded in the organization's culture and memory" (p. 1262). In our view, this fragility is linked to the specific features of temporary organizations. Complex industrial projects, in particular, bring together 
different skills and specific working practices. Hence, in settings that are characterized by disorganization and organizational differences, it appears that organizational or individual sources of resilience are not fully applicable (Milch \& Laumann, 2016). Furthermore, in the face of increased inter-organizational complexity and unstable work processes, Milch and Laumann (2016) show that it is more difficult to build, maintain and develop steady interrelations and knowledge. They highlight the phenomenon of dilution of competences, which arises from the fact that contract workers are unfamiliar with the local work environment and lack industry-specific knowledge and experience. The challenge is how to build and sustain these skills and expertise in the face of temporariness and fragmentation.

We argue that the resilient capability of complex projects relies not only on their use of forms of temporary organizing but also on the occupational groups who develop long-term and inter-organizational skills. Consequently, deepening our understanding of the role these groups play may improve our knowledge of how this particular organizational form is able to maintain a high level of safe performance.

\section{Coordination Between Occupational Groups as a Source of Project Resilience}

By focusing on the occupational dimension, our approach is in line with the practice-based view of safety and reliability (Gherardi, 2018; Gherardi \& Nicolini, 2000; Tillement et al., 2009). Through the practice lens, safety and resilience are seen as a collective knowledgeable doing [that emerges] from the working practices of a community" (Gherardi, 2018, p. 12). Following the concept of communities of practice (Brown \& Duguid, 1991; Lave \& Wenger, 1991; Wenger, 2010), these authors highlight the role of occupational identity in developing resilient capabilities to deal with unexpected situations. Bourrier (1996) identifies coordination processes between occupational groups as being one of the main success factors for dealing with complex and risky events such as outages in nuclear power plants. Her analysis also shows the importance of the coordination of scheduling and carrying out maintenance work. However, her approach to coordination is based more on a strategic analysis of power relationships between groups than on professional cultures, knowledge and expertise. 
Taking the case of a modernization project by a rail company, Tillement et al. (2009) highlight the limitations of written procedures for reacting to incidents and insist on the importance of the situated and sophisticated skills developed by each occupational group, which are based on practical and relational knowledge mediated by discursive and material artefacts such as plans or installations. Observing two different teams, they show that the resilience of the project's organization is supported by occupational communities that are able to deal with unexpected events by developing flexibility through sharing a "coherent vison of the work to be done and the methods to be used" (p. 246) and by identifying the most competent member to solve the problem. However, in Tillement et al.'s (2009) case, if resilience is enhanced inside each team, different "occupational groups have divergent representations concerning the nature of occupations and on the appropriate risk control practices" (p. 250); indeed, sharing practices across boundaries is a key issue in inter-organizational projects.

Kellogg et al. (2006) note that coordination across occupational boundaries is difficult because "expertise and interests [are] 'at stake' for community members" (p. 26). They demonstrate how the company members they observed develop coordination practices, similar to Galison's (1999) concept of a 'trading zone'. In this perspective, 'enacting a trading zone does not require equivalence or similarity of interpretations or interests [...] Instead, members of different communities coordinate their actions temporarily and locally, navigating their differences in norms, meanings and interests only as needed' (Kellogg et al., 2006, p. 39). In the same vein, Bechky (2006) shows that in temporary organizations, coordination between the various stakeholders is permitted through a role structuration, where individuals play a predetermined role and adapt their attitude to the particular situation they face. This coordination ability, based on dual behaviour which combines occupational belonging and the ability to adapt, appears to be a condition for ensuring resilience in temporary projects.

\section{MeTHODS}

To understand how temporary organizing and occupational groups mutually support organizational resilience, we draw on an on-going longitudinal case study of a particular occupational group - the boilermakers-in a naval construction project, an activity which is carried out both by 
internal teams belonging to the principal company and by teams of contractors. The principal company is a shipyard. It is an old semipublic company whose activities and funding depend to a large extent on governmental decisions. We chose the boiler-making activity for two reasons. Firstly, the activity, which mainly consists of installing the pipes in the vessel, is key to the safety of the ship in the medium and long terms. The quality of the installation and the welding are critical to ensuring safety and avoiding the occurrence of major accidents. Secondly, as boiler-making work is fragmented into many operations and between various teams and companies, managing co-activity with other occupational groups (e.g. painters, mechanics or electricians) is essential but very complex. Since co-activity and complexity increase the possibility of unexpected events happening (LePlat \& Faverge, 1967; Perrow, 2011), resilience is crucial for carrying out the activity efficiently and safely.

\section{Data Collection}

We draw on the data collected by one author who was involved from June 2018 to July 2019 in a major research programme, which was carried out in two main phases. In the first phase of the study, she met with several middle managers in the department in charge of installing all the pipes in the vessel and she observed coordination meetings. She also conducted interviews to better identify the safety and industrial issues they were dealing with. This first phase allowed her to obtain a global understanding of the construction project and to more clearly identify the type of complexity involved in the project. During the second phase, she successively followed three teams of boilermakers who were employed by three different companies. Two were subcontractors and the third was a team employed by the principal company which was responsible for the whole construction project. She spent five days with each team, following their shift schedules and shadowing them (Journé, 2005; McDonald, 2005) on the construction site. This enabled her to identify how they worked and organized themselves, which difficulties they were facing and which other occupational groups they interacted with. She completed her observation through individual semi-structured interviews with team members in order to collect more information about their professional background and experience. Interviewees were selected in such a way as to obtain a representative sample which would reflect the various profiles and the divergent views of the global organization. Finally, in order to validate 
Table 4.1 Data collection summary

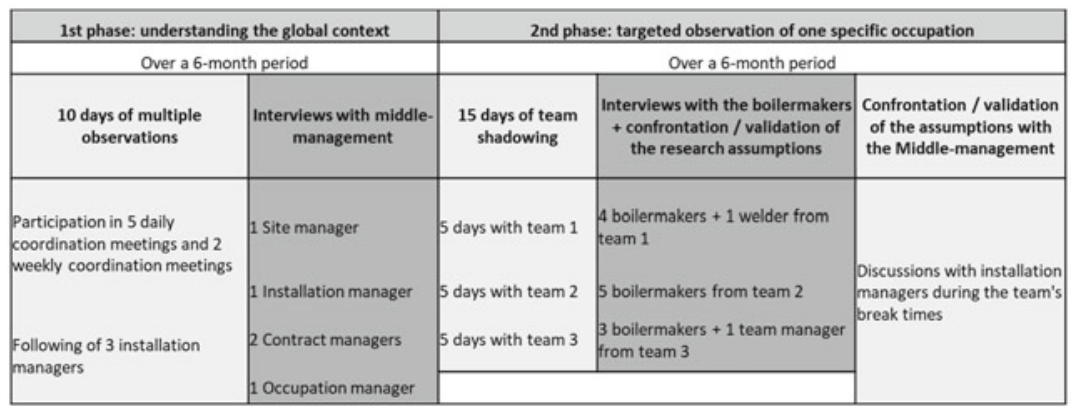

her research assumptions, she presented them to the workers during the interviews and subjected them to the workers' views. This allowed her to consolidate and adjust her findings. She also discussed her hypotheses with the company's middle management (Table 4.1).

\section{Data Analysis}

The researcher's observations were all documented in field notes and the interviews were fully transcribed. She categorized these data manually in order to evidence the role that the principal company and the boilermakers each played in project resilience. The field notes constitute the primary source of information, and they helped to clarify the organizational context in which the project took place and to identify the articulation issues between the organization's formal rules and the boilermakers' occupational practices. They were useful for describing how the various groups of actors worked, coordinated and communicated with each other. The field notes were complemented by the interviews, which provided more information about the actors' motives and preoccupations. They were essential for understanding why middle management and team workers behave differently and do not share values and habits across organizations. They also helped to correct certain assumptions that stemmed from the observation phase. Finally, discussing her assumptions with the boilermakers and the installation managers helped the researcher to refine her classification work and to distinguish between what was shared by members of the team and occupation and what was more specific to an individual or a restricted group. 


\section{EMPIRICAL FINDINGS}

This section shows the role of three main actors and their respective practices in enhancing resilience. The actors are the project organization, the occupational groups and the installation managers. Each actor contributes to resilience through specific processes. First, the project organization has designed an adapting structure in which the actors are prepared to permanently adapt to changes and evolutions in the shipyard's organization. Second, the occupational groups maintain a high level of expertise in a sustainable way. Finally, the installation managers articulate the project constraints with the occupational working practices. These results provide the answers to the three questions posed in the Introduction to this chapter.

\section{Achieving Resilience by Adapting the Workforce to Carry Out a Technologically and Organizationally Complex Project}

The naval construction project studied here presents a huge technological challenge for the principal company responsible for its completion. The project involves building a series of vessels whose technology is completely new and unique. The company last undertook such an ambitious project several decades earlier. At that time, building vessels with high levels of industrial and information technology was the company's core activity, and it recruited a large number of employees in professions and occupations required by the construction projects. Many employees were manual workers involved in building and assembling the various components of the vessels. They belonged to a variety of professional occupations: painting, boiler-making, electrical work, welding, etc. The different occupations required for the various construction steps are shown in Table 4.2 .

Most of the workers were permanent employees of the principal company, which also relied on outsourcing for small parts of the project. However, decades later, when the new project began, the company had lost many manual competencies; as its core activity now relates more to new technological issues, the number of manual workers employed has reduced considerably. The company was therefore facing two major challenges. Firstly, as its internal workforce had progressively moved to ship repair and maintenance tasks, it had lost some of its occupational expertise and its ability to build entire ships. There was also a lack of competent 
Table 4.2 Professional occupations and activities

\begin{tabular}{|c|c|c|c|c|}
\hline & $\begin{array}{l}\text { Fabrication } \\
\text { Department }\end{array}$ & $\begin{array}{l}\text { Installation } \\
\text { Department }\end{array}$ & $\begin{array}{c}\text { Logistics } \\
\text { Department }\end{array}$ & $\begin{array}{c}\text { Quality } \\
\text { Department }\end{array}$ \\
\hline PIPEWORK & Pipe workshop & $\begin{array}{l}\text { Boilermakers } \\
\text { Welders }\end{array}$ & Pipe warehouse & $\begin{array}{l}\text { Pipe quality } \\
\text { controllers }\end{array}$ \\
\hline STRUCTURE & $\begin{array}{l}\text { Structure } \\
\text { workshop }\end{array}$ & $\begin{array}{l}\text { Shipwrights } \\
\text { Mechanics }\end{array}$ & & \\
\hline ELECTRICITY & Wire workshop & Electricians & & \\
\hline \multirow[t]{2}{*}{ PAINTING } & & Painters & & \\
\hline & & $\begin{array}{c}\text { Safety } \\
\text { Department }\end{array}$ & & \\
\hline \multicolumn{2}{|c|}{ CROSS-FUNCTIONAL ACTIVITIES } & Firemen & Storekeepers & Supervisors \\
\hline
\end{tabular}

and experienced managers who were capable of leading large construction projects. Secondly, as the number of manual workers had considerably reduced, the company now had to rely on a large number of contractors and subcontractors to help it meet its deadlines. The contractors thus play a new role: they do not provide the lost expertise which was previously available inside the company; instead, they help the company to re-acquire the expertise that it has lost but that the contractors have maintained. The contractors are therefore also a source of training for the company, which is able to learn from the contractors' experience in different industrial settings. Outsourcing thus constitutes a form of temporary organizing (Söderlund, 2000) which directly contributes to a first component of project resilience: its ability to meet fixed objectives, i.e. building a series of vessels.

The complexity of the naval project lies in its volatility: the activities on the construction site are constantly changing in such a way that no two days are alike. This also means that as each vessel progresses through the construction process, some stages require the presence of certain occupational groups that were needed less in earlier stages or will be needed in subsequent stages. For instance, the role of the shipwrights is crucial for building the overall structure of the ship, but once the project reaches the assembling stage, the shipwrights are less present. As in many projects, the principal company thus has to adapt the workforce to the demands of the 
project. A solution was to engage contracting and subcontracting companies which could provide a temporary workforce when needed. This pool of adaptive resources thus directly contributes to the performance of the project, as they create a continually available and adequate workforce to do the necessary work without exceeding the estimated budget.

We also observed that the projects employed different types of outsourcing practices to meet its organizational needs, which we categorize into two types: long-term contractors and occasional contractors. The former are those who have worked on the ship construction project for a long time. They have developed a good knowledge of the organizational rules and of the way the project is structured and led. As they collaborate on a regular basis with the principal company, they tend to be responsible for project tasks that last for several months. The occasional contractors do not work for the project on a regular basis and constitute a more heterogeneous group of workers. Some of them only work on the project for a few days, while others come and go, spending a few weeks or months at the construction site each time. Their knowledge of the project is thus more limited and so the principal company cannot rely on them to the same extent. However, the occasional contractors are also a necessary workforce for the project because they help the principal company to cope with the many issues and unexpected events that are inherent in technically complex industrial projects: manufacturing errors, changes of schedule, plan modifications, on site installation problems, etc. Using temporary forms of organizing that complement the permanent organization appears to enhance project resilience, allowing it to adapt the required workforce to the production flow of the project.

\section{Occupational Groups Contributing to Resilience by Maintaining Operational Expertise Throughout the Project}

The teams of boilermakers we studied belong to one community which share common practices and values and have the same vision of how a good job should be carried out. In this section, we first show why the boilermakers constitute a community of practice in the sense developed by Lave and Wenger (1991), where they are united around a shared domain of interests, the same community and a common set of practices. We then highlight how these characteristics contribute to the project's performance and safety: by enhancing its flexibility and reactivity, the community contributes to the project's overall performance, 
and, by developing a deep sensitivity to practice, it acquires a situated understanding of occupational and industrial safety issues.

The three teams of boilermakers we studied belong to three different companies and work under different contracts: some of them are directly employed by the principal company (team C), others are contractors (teams $\mathrm{A}$ and $\mathrm{B}$ ) and $\mathrm{a}$ few are subcontractors who are temporarily employed by a contractor (teams A and C). They also have different levels of knowledge of the project, different kinds of previous experience and different occupational backgrounds. Despite all these differences, the boilermakers are part of an occupational community in the sense that they share a set of common values and all agree about what constitutes good boiler-making and how it should be done. For them, boiler-making is first and foremost manual work, which they learn through observation and practice. They consider boiler-making to be precision work that has to follow an ordered process and requires time to be done correctly. In addition, they have formed a physical community which gets together during and outside of working hours and which has created an occupational network beyond the organizational and project boundaries.

The boilermakers also have a shared domain of interest-boilermaking-which, in this project and in the particular context of marine infrastructure, is the activity of assembling, installing and fixing the pipes in the vessel. Many of them chose to become boilermakers because they were good with their hands and enjoyed the material dimension of the job. They have developed a particular boiler-making vocabulary, sometimes using nicknames to refer to particular tools and materials, and they have developed certain procedures that are specific to the boiler-making activity.

Finally, the boilermakers share a common set of practices, which we observed in the three teams studied. A good example of this is the way each team is structured, following the same hierarchical organization. At the top, the team manager is responsible for the contractual link with the principal organization and for coordinating the team's work progress. Each team is divided into sub-groups, led by senior boilermakers who coordinate the work and are responsible for ensuring that the installation complies with the principal company's assembly plan; they take responsibility if any errors are detected. Also, in each team the boilermakers work in pairs, usually composed of a senior and a more junior worker. 
They have also developed the same habits and routines: when installing a pipe, they generally follow the same steps, i.e. examining the installation plan, measuring the location where the pipes have to be installed, loading the pipes, making adjustments when needed, and finally installing the pipes with the help of the welder. To do their work efficiently, they also rely on the same tools, which they carry in one of the many pockets of their blue overalls or in their leather case. These include a pencil, a small notebook, a tape measure, different sizes of wrenches, etc., and their mobile phone calculator app. In the following paragraphs, we will see how this community functioning enables the boiler-making group to contribute to the project's efficiency and to meet the expected deadlines.

Firstly, the occupational teams are structured in a way that make them flexible and enable them to reorganize quickly when faced with unexpected changes. They are led by senior experts whose technical legitimacy is recognized and respected. This enables the teams to react more thoughtfully and quickly when a problem comes up. This formal organization, similar in the three teams, favours collective decision-making based on technical expertise. Indeed, if there is any doubt or disagreement, the decision will often be taken by the more experienced worker. Furthermore, their organization remains flexible and can be changed according to the situations that arise. For example, when a particularly technical task is to be carried out, the manager can decide to pair two senior workers so that he can be sure that the work will be done perfectly. So, the boilermaker teams have a top-down, but not rigid, organization: as the project progresses and new situations arise, they can redesign their organization and adapt in a flexible way that contributes to the project's resilience.

The teams' efficiency and ability to deal with unexpected situations also rely on strong leadership, embodied by the team manager or by the section managers, who generally have previous boiler-making experience and who are respected and trusted by the other members of the team. This leadership is essential for the team's resilience: when the team faces unexpected situations or great pressure, the team leaders play a key role in encouraging the other workers and in achieving their shared goal.

Their work follows organizational routines which structure their activity and give them the opportunity to share their respective knowledge and experience. For example, we observed that the teams have a daily routine of taking collective breaks. These breaks are convivial times when the workers talk about their personal lives, make jokes or complain about their work problems, depending on the mood of the day. These moments 
have a direct impact on their ability to work together efficiently. Because they are moments of sharing, they help to reinforce the team spirit and are opportunities for getting to know each other better. This reinforces the workers' ability to trust each other when they are working together in a risky context.

Finally, the boiler-making community shares common values, attitudes and concerns about their occupation and its risks which transcend the organizational boundaries while ensuring a high level of industrial safety. A first challenge for the workers when they arrive on site is therefore to learn to adapt to the constant changes without putting themselves in danger. To avoid accidents, the workers develop a sense of awareness and learn to always be careful to look out for any structural changes. For instance, when a boilermaker arrives in a particular section of the site, he looks at the area and checks if there have been any changes since he was last there. Likewise, he will be very careful about where he puts his feet; if a tool, a cable or an air duct is lying on the floor, he will systematically take it away and try to hang it on a scaffold.

Another major preoccupation of the practitioners is industrial safety, which ensures that their work is safe and under control. By establishing routinized checking procedures and by continually practising these, they develop a more situated and embedded understanding of the potential risks. As different occupations can be working on the same line of pipe at the same time, the workers have to deal with work fragmentation. In this context, checking procedures ensure global coherence, which has a direct impact on the industrial safety of the installation process. The boilermakers' technical expertise also lies in their ability to use the various tools they need in their daily activity. However, practice is essential to acquire some skills, and takes time. For instance, it takes at least six months for a beginner to be able to correctly chamfer a pipe, and the boilermakers believe it takes 10 years to become a senior boilermaker and 15 or 20 years to be an expert. It is also through practising that the workers learn which tools are more appropriate to ensure a better quality of installation, which directly contributes to industrial safety.

To conclude, the boilermakers constitute a community of practice which develops an in-depth understanding of the project's risks and constraints. This contributes to the project's resilience by ensuring a high level of performance while maintaining occupational and industrial safety. 


\section{Coordinating Working Practices in Temporary Organizing Contexts: Support for Project Resilience}

We have seen that the boiler-making activity in this naval project is composed of different teams belonging to different companies, with boilermakers working under different work contracts. This diversity offers the project some flexibility because it enables the principal company to continually adapt the number of workers in the worksite according to its evolving workload. At the same time, to deal with the different rules and working practices between the various teams of boilermakers, the company has set up coordination mechanisms that bring together the different teams of workers around the same priority: avoiding co-activity constraints. These coordination mechanisms take the form of weekly and daily meetings attended by representatives of the various occupational groups co-operating on the shipyard, and we show how they help to prevent unexpected events and contribute to the workers' safety.

The purpose of the weekly coordination meeting, which gathers together representatives of all the occupations and teams working in a specific ship section, is to monitor the schedule. At this meeting, the participants review the work to be carried out each day in each sector by each occupational team. The manager of each team confirms whether the planned tasks can be done or should be rescheduled. The aim of the meeting is to avoid co-activity whenever possible and to articulate the work between the planned and the actual activity, which reduces loss of time and enhances the workers' efficiency. However, if an unexpected event happens, the team cannot wait until the next weekly meeting to solve the problem and a daily coordination meeting has therefore also been put in place.

The purpose of the daily coordination meeting is to adjust the planned work to the situation on site. The daily meeting thus enables greater situated management of the co-activity between the various occupational groups by creating space for informal work discussions that overlap with the formal meeting (box 1). They provide an important opportunity for the managers to make arrangements and find practical solutions for their teams. 


\section{Box 1: Informal talks during a coordination meeting}

As the coordination meeting drags on, two managers in charge of two different teams of boilermakers start a discussion on co-activity issues in their sector:

Manager A: When will you finish the welding on pipeline B32? We really need to start the installation on B33.

Manager B: This afternoon, I hope, but it depends on the air ducts. I'm not sure that Logistics have activated them. Always the same problem...

M A: How many of you are there just now?

M B: Three with the welder.

M A: Ok. There can be six of us. So maybe my guys could start the measurements right now.

$\mathrm{M} \mathrm{B}$ : Ok, my guys are cool so no problem if your guys work in the same sector.

M A: Great.

By setting up various coordination meetings, the principal company can better anticipate the frequent organizational changes that are inherent in complex industrial projects and find collective solutions when blocking points arise (box 2).

\section{Box 2: Coordination meetings managing occupational issues}

\section{Tuesday:}

8:30: The Installation Manager meets the Team Manager in the team workspace. The Team Manager informs the Installation Manager about a pipe-installation issue: a piece of pipe is too long and needs to be recut on site because it has already been welded. This problem has held up the team since the beginning of the morning.

8:45: The Installation Manager and the Team Manager go and see the pipe on board. They realize that this operation risks damaging the painting. Moreover, a scaffold is preventing the boilermakers from cutting the pipe. The Installation Manager needs 
the opinion and authorization of the Section Manager to take a decision.

9:00: The Installation Manager participates in the daily coordination meeting and shares the pipe issue with the other members. For the Section Manager, the priority is to reach the next milestone: he advocates for the quicker solution, which seems to be cutting the pipe on board. The Installation Manager in charge of the painting is worried about the potential damage. He suggests the boilermakers use a saw instead of a grinder to cut the pipe. Finally, the Installation Manager in charge of the scaffolder team plans an intervention in the morning.

9:30: End of the daily meeting. The Installation Manager calls the Team Manager and tells him they can cut the pipe with a saw once the scaffolds are removed.

10:30: The scaffolders go on board and rearrange the scaffolds.

11:00: The boilermaker in charge of cutting the pipe arrives on board and realizes he doesn't have a saw. It takes him almost an hour to find the right one.

13:00: End of the working day for the boiler-making team. The boilermaker hasn't finished the work.

Wednesday:

9:30: The boilermaker finishes cutting the pipe with the saw. He informs the Team Manager, who remarks that the event has caused a delay to the schedule.

10:00: The Team Manager shares the information with the Installation Manager who has just left the daily meeting. The Installation Manager informs the Coordination Manager, who makes a change to the schedule.

The second role of the weekly meeting is to ensure the occupational safety of the workers by defining the organizational conditions that enable safe co-activity. For example, when the boilermakers and the welders are working together, the painters may be prevented from working because the welding sparks will damage the painting. In this case, the solution may be to install a temporary separating wall so that both occupations can work at the same time without any risks. These coordination practices thus contribute to the progress of work on the construction site; they 
allow the project to be more resilient by improving its overall performance and by anticipating potential accidents.

\section{Discussion AND Conclusion}

\section{Resilience in the Tension Between Permanent and Temporary Forms of Organizing}

Researchers have emphasized that new forms of organizing tend to be more fragmented and temporary (Kellogg et al., 2006) in order to enhance flexibility and performance. However, most works have studied resilience in long-standing organizations from a systemic perspective. They have shown that both anticipation and adaptation are supported by strong organizational routines (Hollnagel et al., 2006). Even if the literature on temporary organizing and the literature on resilience are rarely articulated, they emphasize near-opposite views of the foundations of resilience: flexibility on the one hand, stability on the other hand.

Based on this observation, we seek to combine these two research areas by studying how resilience can be expressed and enhanced in contexts where temporary forms of organizing articulate with the sustainable structure of occupations. We study the construction of a new series of ships by a shipyard, where occupational groups, notably the boilermakers, are temporarily involved and gathered together in a project-based organization. Taking a dynamic approach, we define temporary organizing as a complex and always evolving mix of temporary and permanent elements. This perspective enables us to make three theoretical and methodological contributions.

First, unlike the cases studied by Bourrier (1996) and Tillement et al. (2009), the project under study is fundamentally a temporary structure and does not juxtapose with a larger routinized or bureaucratic organization. Moreover, every worker is a temporary worker in this project-based organization. Thus, the challenge for resilience is not to articulate the permanent and temporary organizational structures, but to articulate a temporary project organization with more permanent occupations. In the cases studied by Bourrier (1996) and Tillement et al. (2009), organizational resilience is threatened by the rivalries between historical occupational groups that are representatives of the permanent structure and the emerging ones that are representatives of the projectbased organization. Our case differs from theirs and highlights another 
configuration: since all the workers are involved temporarily in a transient ship construction project, there are no major conflicts or rivalries between the various occupational groups. All the workers are gathered around the same object, the ship, which plays the role of a boundary object (Bechky, 2006; Leigh Star, 2010). In our case, the tensions do not lie at the interoccupational level but, rather, at the inter-organizational level. We observe tensions which are associated with power relationships between the boilermakers of the principal company and the contractors, and symbolic dimensions: the former consider themselves as more legitimate, with the latter being seen as a secondary workforce. In this particular organizational configuration, the relations between occupational groups, which favour robust coordination mechanisms, support resilience rather than constituting vulnerability.

Second, we show that resilience is also grounded within each community of practice, which transcends organizational boundaries and thus contributes to the project's overall coordination ability. The community of boilermakers we observed is similar to the communities described by Brown and Duguid (1991), who define them as "more fluid and interpenetrative than bounded, often crossing the restrictive boundaries of the organization to incorporate people from outside" (p. 49). Similarly, the boilermakers' occupational groups are constantly evolving. The arrival of newcomers and the discussions and sharing of various experiences help to enrich their knowledge and expertise. This learning process (similar to apprenticeship) is embedded in the legitimate participation of the members of the community of practice (Lave \& Wenger, 1991) and thus is conditioned by the possibility of the actors being fully integrated into the community's social life and sharing its occupational values.

Finally, from a methodological point of view, our study highlights the value of adopting a meso-level of analysis to study the practices and processes that enhance resilience. Surprisingly, this level of analysis is rarely used either in the temporary organizing literature (Bakker et al., 2016) or in the resilience engineering's perspective, which has mainly focused on the organizational or individual dimensions. Our group-level analysis enables us to show in greater detail how the various communities of practice manage to coordinate their activity, and to highlight the role of discussions and inter-group communication in dealing efficiently with unexpected situations. This demonstrates in a more situated and deeper way the organizational mechanisms of resilience by describing the concrete adaptation and reaction practices developed to deal with 
unexpected situations. From a longer term perspective, this practice-based view of resilience emphasizes the role of occupations in supporting longlasting learning dynamics, which are able to go beyond the temporary forms of organizing that lie both in the project structure and in workers' participation.

\section{Managerial Contributions}

In safety-critical projects such as the one under study, resilience partly relies on the involvement of key individuals who perform an interfacing role between project managers and occupational groups. However, frequent turnover hinders the ability of the workers to build sustainable resilient practices. Moreover, the frequently changing structure of the project organization tends to result in disengagement from the long-term goals. It is thus crucial to reflect upon the organization's ability to build long-lasting learning dynamics that can ensure enduring organizational resilience. We propose two conditions for the organization to maintain its resilient capacity in the long term: keeping key individuals in lasting positions and retaining key competences throughout the project.

First, coordination between the occupational groups and project management mainly relies on the key role of the installation manager and on his ability to interact efficiently with the team manager. As the installation manager needs time to acquire his coordination ability and to become familiar with the technicalities of the boilermakers' work, staying longer in his position could contribute to enhancing resilience in the longer term. This observation reveals a major discrepancy between the perspective of the project managers, whose goal is to use this position to educate the newcomers about the site constraints before they join the field offices, and the perspective of the workers, who need a single and reliable interlocutor to respond efficiently to the project managers' requests.

Secondly, as contract workers possess rare competences, they can easily negotiate a better salary or a better position with the highest bidder. In this context characterized by high levels of competition, the principal company must develop a competence retention strategy to avoid 'losing' the more competent workers. Workers give two main reasons for leaving a project: the feeling of not being involved in the whole project and the lack of technicity and diversity of the tasks. Thus, involving contract workers in the global project and informing them about the overall contribution of their work is making to the project are essential for project resilience. This 
entails building partnership relations with contractors, based on longterm contracts and on shared training programmes. The ability of the project to retain competences also relies on the workers sharing their knowledge and training in order to become multi-skilled experts. In a time-pressured context of occupational distribution, the experts lack the time to train the beginners. As a consequence, we recommend that the work of the occupational groups should be considered as a global process of legitimate peripheral participation in communities of practice (Lave, 1991).

\section{Limitations and Avenues for Further Research}

In our study, we focused on one specific occupational group, i.e. the boilermakers, which enabled us to highlight foundations of resilience linked to learning dynamics (similar to apprenticeship) that enable the development and maintenance of shared and situated practices. For future research, it would be interesting to study another occupational group, such as electricians, in order to test the generalization of our results on a larger scale. In addition, studying the practices of different occupational groups in parallel might enable a better understanding of the basis of inter-occupational coordination. Finally, as we paid little attention in this study to contractual agreements and their effects on coordination, this could merit further study. Finally, our case focused on a complex project that was led by a semi-public nature organization, which contracted out activities to private companies. To better qualify the effects of the degree of publicness (Bozeman \& Bretschneider, 1994) on project resilience, it could be interesting to compare this particular case with other cases where projects are led by private companies.

\section{REFERENCES}

Bakker, R. M., DeFillippi, R. J., Schwab, A., \& Sydow, J. (2016). Temporary organizing: Promises, processes, problems. Organization Studies, 37(12), 1703-1719.

Bechky, B. A. (2006). Gaffers, gofers, and grips: Role-based coordination in temporary organizations. Organization Science, 17(1), 3-21.

Bourrier, M. (1996). Organizing maintenance work at two American nuclear power plants. Journal of Contingencies and Crisis Management, 4(2), 104112. 
Bozeman, B., \& Bretschneider, S. (1994). The "publicness puzzle" in organization theory: A test of alternative explanations of differences between public and private organizations. Journal of Public Administration Research and Theory, 4(2), 197-223.

Brown, J. S., \& Duguid, P. (1991). Organizational learning and communitiesof-practice: Toward a unified view of working, learning, and innovation. Organization Science, 2(1), 40-57.

Furuta, K. (2015). Resilience engineering. In J. Ahn, C. Carson, M. Jensen, K. Juraku, S. Nagasaki, \& S. Tanaka (Eds.), Reflections on the Fukushima Daiichi nuclear accident: Toward social-scientific literacy and engineering resilience (pp. 435-454). Springer.

Galison, P. (1999). Trading zone: Coordinating action and belief. The Science Studies Reader, 13, 137-160.

Garfinkel, H. (1967). What is ethnomethodology? In H. Garfinkel (Ed.), Studies in ethnomethodology (pp. 1-34). Prentice-Hall.

Gherardi, S. (2018). A practice-based approach to safety as an emergent competence. In C. Bieder, C. Gilber, B. Journé, \& H. Laroche (Eds.), Beyond safety training: Embedding safety in professional skills (pp. 11-21). Springer.

Gherardi, S., \& Nicolini, D. (2000). The organizational learning of safety in communities of practice. Journal of Management Inquiry, 9(1), 7-18.

Hollnagel, E. (2016). Resilience engineering: A new understanding of safety. Journal of the Ergonomics Society of Korea, 35(3), 185-191.

Hollnagel, E., Woods, D., \& Leveson, N. (2006). Resilience engineering: Concepts and precepts. CRC Press.

Journé, B. (2005). Etudier filemanagement de l'imprévu: Méthode dynamique d'observation in situ. Finance Contrôle Stratégie, 8(4), 63-91.

Kellogg, K. C., Orlikowski, W. J., \& Yates, J. (2006). Life in the trading zone: Structuring coordination across boundaries in postbureaucratic organizations. Organization Science, 17(1), 22-44.

Lave, J. (1991). Acquisition des savoirs et pratiques de groupe. Sociologie Et Sociétés, 23(1), 145-162.

Lave, J., \& Wenger, E. (1991). Situated learning: Legitimate peripheral participation. Cambridge University Press.

Leigh Star, S. (2010). This is not a boundary object: Reflections on the origin of a concept. Science, Technology, \& Human Values, 35(5), 601-617.

LePlat, J., \& Faverge, J. M. (1967). Recherche communautaire sur la sécurité dans les mines et la sidérurgie: Étude no. 3/6, recherche dans les mines de fer français (Community research on safety in mines and the steel industry: Study No. 3/6, Research in French iron mines). Doc. No. 8085/65 f, 1967.

Lundin, R. A., \& Söderholm, A. (1995). A theory of the temporary organization. Scandinavian Journal of Management, 11(4), 437-455. 
McDonald, S. (2005). Studying actions in context: A qualitative shadowing method for organizational research. Qualitative Research, 5(4), 455-473.

Milch, V., \& Laumann, K. (2016). Interorganizational complexity and organizational accident risk: A literature review. Safety Science, 82, 9-17 (2016).

Perrow, C. (2011). Normal accidents: Living with high rish technologies (Updated). Princeton University Press.

Saunders, F. C. (2015). Toward high reliability project organizing in safetycritical projects. Project Management Journal, 46(3), 25-35.

Saunders, F. C., Gale, A. W., \& Sherry, A. H. (2016). Responding to project uncertainty: Evidence for high reliability practices in large-scale safety-critical projects. International Journal of Project Management, 34(7), 1252-1265.

Söderlund, J. (2000). Temporary organizing-characteristics and control forms. In R. A. Lundin \& F. Hartman (Eds.), Projects as business constituents and guiding motives (pp. 61-74). Springer.

Tillement, S., Cholez, C., \& Reverdy, T. (2009). Assessing organizational resilience: An interactionist approach. M@n@gement, 12(4), 230-264.

Van Maanen, J. (1979). The fact of fiction in organizational ethnography. Administrative Science Quarterly, 24(4), 539-550.

Weick, K. E. (1979). The social psychology of organizing (second edn.). McGrawHill. Reviewed by K. E. Weick in M@n@gement, 2015, 18(2), 189-193.

Weick, K. E., \& Roberts, K. H. (1993). Collective mind in organizations: Heedful interrelating on flight decks. Administrative Science Quarterly, 38(3), 357-381.

Weick, K. E., \& Sutcliffe, K. M. (2015). Managing the unexpected: Sustained performance in a complex world (3rd Edition). John Wiley \& Sons.

Wenger, E. (2010). Communities of practice and social learning systems: The career of a concept. In C. Blackmore (Ed.), Social learning systems and communities of practice (pp. 179-198). Springer. 
Open Access This chapter is licensed under the terms of the Creative Commons Attribution 4.0 International License (http://creativecommons.org/licenses/ by $/ 4.0 /$ ), which permits use, sharing, adaptation, distribution and reproduction in any medium or format, as long as you give appropriate credit to the original author(s) and the source, provide a link to the Creative Commons license and indicate if changes were made.

The images or other third party material in this chapter are included in the chapter's Creative Commons license, unless indicated otherwise in a credit line to the material. If material is not included in the chapter's Creative Commons license and your intended use is not permitted by statutory regulation or exceeds the permitted use, you will need to obtain permission directly from the copyright holder.

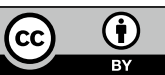

